\title{
Application value of real-time shear wave elastography in differential diagnosis of testicular torsion
}

\author{
Ensheng Xue ${ }^{1,2}$, Yue Yu ${ }^{1}$, Lulin Lin', Zhiyong Li', Huanzhong Su' \\ ${ }^{1}$ Department of Ultrasound, Fujian Medical University Union Hospital, ${ }^{2}$ Department of Imaging of Fujian Medical \\ University, Fuzhou, China
}

\begin{abstract}
Aim: To evaluate the clinical value of real-time shear wave elastography (SWE) in differential diagnosis of testicular torsion and acute orchiditis. Material and methods: During a 3-year period, 14 cases of testicular torsion and 16 cases of acute orchiditis met the inclusion criteria. Young's modulus maximum hardness (Emax) of testicular capsule region, middle testicular parenchyma, warped spermatic segment or inferior spermatic segment was measured in each group. SWE "stiff ring sign" of testis refers to the appearance of a red ring in the testicular capsule area and "stiff knot sign" of spermatic cord refers to the appearance of a red knot in the lower segment of the spermatic cord. Results: Emax value of the testicular capsule in the torsion group was higher than in the acute inflammation group $(138.76 \pm 58.27 \mathrm{vs} 16.40 \pm 4.71 \mathrm{kPa}, \mathrm{p}=0.0001)$. Emax value in the middle parenchyma of the testis showed no statistically significant difference between groups $(\mathrm{p}=0.053)$. Emax value in the twisted spermatic segment was higher than that in the lower spermatic segment with acute inflammation $(166.61 \pm 60.07$ vs $14.14 \pm 4.93, \mathrm{p}=0.0001)$. In the torsion group, 12 testicular capsule areas showed "stiff ring sign" and all twisted segments of spermatic cord showed "stiff knot sign" but no signs were found in the inflammatory group. Conclusions: "Stiff ring sign" of testis, "stiff knot sign" of spermatic cord, high stiffness of the testicular capsule and in the twisted spermatic segment are the typical SWE findings of testicular torsion, with important clinical value in the differential diagnosis of testicular torsion and acute orchiditis.
\end{abstract}

Keywords: testicular torsion; differential diagnosis; real-time shear wave elastography; ultrasound

\section{Introduction}

Testicular torsion is a common cause of scrotal emergencies and is often misdiagnosed as acute orchitis and epididymitis because of its atypical clinical manifesta-

Received 13.09.2019 Accepted 11.12.2019

Med Ultrason

2020, Vol. 22, No 1, 43-48

Corresponding author: Ensheng Xue

Department of Ultrasound, Fujian Medical

University Union Hospital

29 Xinquan Road Gulou District,

Fuzhou 350001, China

Phone: +86 591833578968352

Fax: +86 59183339732

E-mail: xuees01@163.com tions [1-3]. Irreversible damage to spermatogenic tissues can occur after 6 hours of testicular tissue ischemia [4]. Therefore, testicular torsion should be diagnosed as early as possible to facilitate testicular survival and protect spermatogenic function $[5,6]$. High frequency color Doppler ultrasonography is accepted to be the primary imaging method used in these cases [7,8]; however, some cases of incomplete testicular torsion can be unrecognized due to atypical sonographic findings $[7,9]$. Contrast-enhanced ultrasound (CEUS) is helpful for the diagnosis of testicular torsion [10-12], but some studies suggest that CEUS has no obvious advantage compared with color Doppler ultrasound [13].

Ophir et al proposed the concept of ultrasonic elastography in 1991, which can provide information about the 
tissues stiffness by detecting their mechanical properties [14]. Shear wave elastography (SWE) is the most cutting-edge ultrasonic elastography technology at present. Compared with strain elastography, SWE has the advantages of quantitative evaluation of soft tissue stiffness, strong repeatability and higher clinical application value [15], including testis pathology [16]. The results of clinical and animal experiments showed that the SWE values of testicular parenchyma significantly increase during testicular torsion $[17,18]$. But it is not clear whether the changes can be distinguished from other scrotum emergencies such as acute orchitis. Testicular torsion is essentially a warping of the spermatic cord; therefore, the SWE performance for other structures of the scrotum, during testicular torsion need to be established.

The purpose of this study was to evaluate the clinical value of SWE in the differential diagnosis of testicular torsion by comparing the SWE findings of testicular capsular area, testicular parenchyma and spermatic cord between testicular torsion and acute orchitis.

\section{Materials and methods}

\section{Patients}

The subjects enrolled for this study were patients who had undergone scrotal ultrasound at the Fujian Medical University Union Hospital from May 2015 through May 2018. Criteria for inclusion were: 1) cases examined by color Doppler ultrasound and SWE; 2) testicular torsion confirmed by operation or; 3 ) diagnosis of acute orchitis established by clinical (testicular swelling and pain, with or without fever) and ultrasound criteria, with conservative treatment and with no abnormal testicular findings at one-year follow-up. In addition, 20 healthy volunteers, with no history of reproductive system diseases and no abnormal findings of scrotal color Doppler ultrasound, were randomly selected for SWE examination. This study was performed with the approval of the local Ethics Committee and written informed consent was obtained from all participants.

\section{Ultrasonography protocol}

The ultrasound examination was performed using $\mathrm{Su}-$ personic Aixplorer (Aix-en-Provence, France) machine with 4-15 MHz linear probe. The frequency used was 12 to $14 \mathrm{MHz}$, the scale of color Doppler is 2 to $4 \mathrm{~cm} / \mathrm{s}$. Subjects were placed in supine position and the scrotum was exposed. The examination started with gray-scale images (echogenicity, homogeneity and size of testis and epididymis together with the relationship between testis, epididymis and spermatic cord were analyzed), followed by color Doppler (testis, epididymis and spermatic cord vascularization was evaluated) and SWE.
The measured value of Young's modulus set to $\geq 100 \mathrm{kPa}$, and the image color ranges from blue, bluegreen, greenish-yellow, yellow-orange to red, representing from soft to hard. The Q-Box ${ }^{\mathrm{TM}}$ was placed in the testicular membrane area (testicular membrane and testicular edge parenchyma within $2 \mathrm{~mm}$ ), in the middle of the testicles and in the warp distortion section or the lower part of the spermatic cord. The Young's modulus Emax (elastography max) of each the above structure was measured. For the same structure, 3 different longitudinal sections were realized, and the Young's modulus was measured once for each section and its average value was taken for further analysis. In the disease group the testicle and spermatic cord of the affected side were measured and in the normal group one testicle and spermatic cord were randomly measured. The size of Q-Box ${ }^{\mathrm{TM}}$ used for evaluation of testicular capsule was $2 \mathrm{~mm}$, of middle testicular parenchyma 5-10 $\mathrm{mm}$, and of torsion segment or lower segment of spermatic cord 10-15 mm. For correct SWE evaluation the sampling box had to be completed filled with color and the SWE artifacts due to probe compression or scrotal roughness were excluded.

SWE "stiff ring sign" of the testicular capsule was defined as a colored "stiff ring" surrounding the testicular edge. SWE " stiff knot sign" of spermatic cord was defined as a colored "stiff knot" locating the twisted lower part of spermatic cord. These signs, a qualitative SWE feature, were evaluated at the display setting of less than $100 \mathrm{kPa}$ and at $100 \mathrm{kPa}$.

Two investigators, with 3 years and above experience in testicular SWE, independently examined all the subjects. The Emax values of testicular capsule area, central testicular parenchyma, torsion segment of spermatic cord or lower segment of spermatic cord in testicular torsion and acute orchitis was determined.

\section{Statistical analysis}

The IBM SPSS software package (SPSS for Windows, version 22.0) was used for statistical analysis. The Kolmogorov-Smirnov normality test of measurement data was carried out first. The data of normality were expressed as mean \pm standard deviation (SD), oneway ANOVA was used for comparison among multiple groups, and Least Significant Difference test was used for pairwise comparison among groups. Median (M) was used for non-normal data, and Mann-Whitney rank sum test was used for comparison between groups. A p value of less than 0.05 was considered to indicate statistical significance. Intraclass Correlation Coefficient (ICC) was used to evaluate the consistency of the results measured by the two examiners. 


\section{Results}

\section{Clinical manifestation}

A total of 30 patients were included in this study, of which 14 had testicular torsion (age 7-48 years, mean $16.79 \pm 12.37$ years, onset time from 4 hours to 28 days) and 16 acute orchitis (aged 16-46 years old, mean age $30.94 \pm 21.82$ years, onset time from 2 days to 14 days). The cases with testicular torsion were operated: 3 cases release surgery and 11 cases testicular resection. Of the 16 cases of acute orchitis, 1 was secondary to bladder perfusion, 3 to mumps and 12 to acute epididymitis. The age of the 20 normal volunteers ranged from 22 to 45 years, with an average age of 29.43(6.41) years.

\section{Ultrasonography of normal testis and spermatic cord}

In the normal control group, the average volume of testis was $9.31 \pm 1.41 \mathrm{ml}$, the testicular parenchyma echo was uniform and normal vascularization was found in all testis. There was no thickening of the terminal spermatic cord, no dilation of blood vessels. Eighteen spermatic cord ends were straight and two were tortuous. On SWE examination, the testicular capsule was uniform blue or blue-green, the central testicular parenchyma and the lower spermatic cord were uniform blue. Emax value in the lower spermatic segment was higher than that in the testicular capsule region and Emax value in the testicular capsule region was higher than that in the middle testicular parenchyma (Table I).

\section{Ultrasound appearance of testicular torsion}

In the testicular torsion group, there were 10 testicular torsion on the left side and 4 testicular torsion on the right side. The testicles had enlarged volume $(14.78 \pm 4.92$ $\mathrm{ml}$ vs $9.31 \pm 1.41$ in normal subjects). The testicular parenchyma was heterogeneous in 11 cases, slight hypovascularization was found in 3 testes, significant hypovascularization in 1 testis and no vascularization in 10 testes. The lower spermatic cord was distorted and swollen in all cases, 6 with "whirlpool" sign, 4 with decreased vascularization and 10 without vascularization.

According to SWE examination, the central parenchyma of all torsion testes showed uneven blue or blue-green color and 12 testicular capsule areas showed yellow-orange or red color, with "stiff ring sign" (fig 1). Twisted segments of all spermatic cord showed orangered or red color, with "stiff knot sign" (fig 2). Emax value in the lower spermatic cord was higher than that in the testicular capsule region and the Emax value in the testicular capsule region was higher than that in the testicular parenchyma (Table I).

\section{Ultrasonographic appearance of acute orchitis}

In the acute orchitis group, there were 11 cases of left testicular inflammation and 5 cases of right testicular inflammation. The testicles showed varying degrees

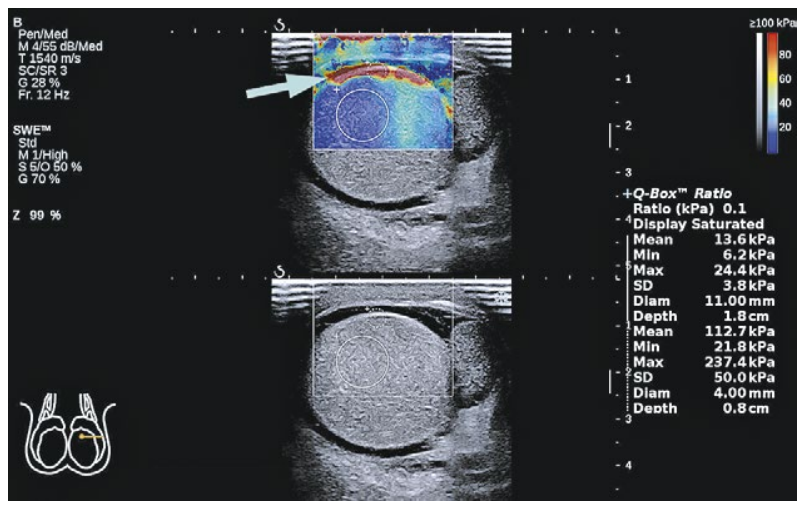

Fig 1. Testicular torsion in a 13 -year-old patient, SWE examination, transverse scan of the left testicle. The testicular capsule showed a curved red color (arrow), Emax value $=237.4 \mathrm{kPa}$

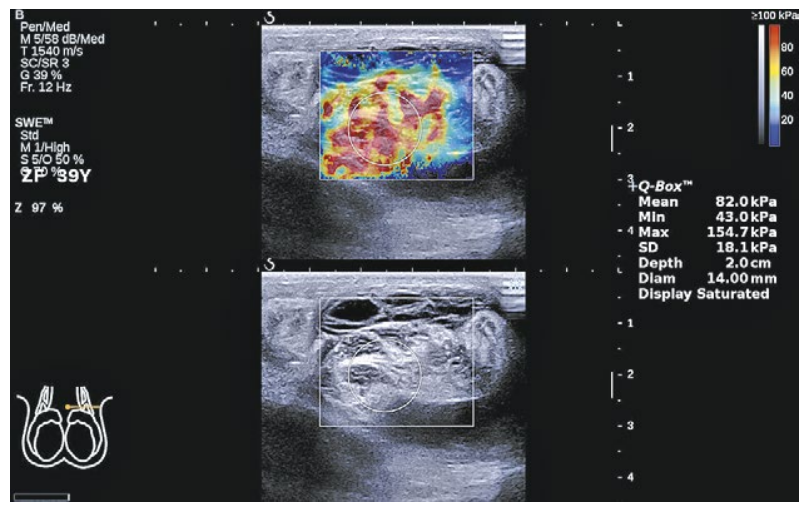

Fig 2. Torsion of spermatic cord in a 43-year-old patient, SWE examination, transverse scan of twisted spermatic cord, showing "stiff knot sign" (arrow), Emax value = $154 \mathrm{kPa}$

Table I. Elastography max measurement results of scrotum structures in each group

\begin{tabular}{|c|c|c|c|c|c|c|}
\hline Group & $\mathbf{n}$ & Testicular capsule & Parenchyma testis & Lower spermatic segment & F value & p value \\
\hline Normal & 20 & $9.73 \pm 2.79$ & $4.51 \pm 1.42$ & $11.45 \pm 2.50$ & 102.514 & 0.0001 \\
\hline Torsion & 14 & $138.76 \pm 58.27$ & $12.39 \pm 6.56$ & $166.61 \pm 60.07$ & 40.262 & 0.0001 \\
\hline Orchitis & 16 & $16.40 \pm 4.71$ & $9.65 \pm 4.85$ & $14.14 \pm 4.93$ & 8.077 & 0.001 \\
\hline $\mathrm{p}$ value & & 0.0001 & 0.0001 & 0.0001 & & \\
\hline
\end{tabular}

$\mathrm{n}$ : number of cases; F: the statistic value of $\mathrm{F}$ test 
of enlargement, with an average volume of $14.78 \pm 4.92$ $\mathrm{ml}$. Four testicular parenchyma echogenicity were homogeneous and 12 heterogeneous. Significant hypervascularization was found in all testes. There were 12 local or whole epididymal enlargement with uneven echo and significant hypervascularization. The lower spermatic cord showed different degrees of thickening, vasodilatation, but without being distorted.

On SWE examination, the parenchyma in the middle of all inflamed testis and lower spermatic cord was uneven blue or blue-green without "stiff ring sign" (fig 3, fig 4). Emax value in the testicular capsule was higher than that in the inferior spermatic cord and Emax value in the inferior spermatic cord was higher than that in the middle parenchyma of the testis (Table I).

\section{Comparison of Emax value of measurements between groups}

Emax values of testicular capsule and in twisted spermatic segment were higher in the torsion group $(p=0.0001)$, Emax values in the middle parenchyma of testis showed no statistically significant difference between torsion and acute inflammation groups $(\mathrm{p}=0.053)$. Emax value in the twisted spermatic segment was higher than that in the lower spermatic segment with acute inflammation ( $\mathrm{p}=0.0001)$, as shown in table I.

\section{Interobserver agreement}

Emax values of scrotal structures independently measured by 2 examinees showed no difference and good consistency, as shown in table II.

\section{Pathologic findings of torsion testis}

Optical microscopy showed the albuginea thickening, interstitial edema and vascular ectasia. The structure of the seminiferous tubule was destroyed, the arrangement of spermatogenic cells was disordered, some or all spermatogonial cells fell off. In severe cases, only residual damaged seminiferous tubule could be shown.

\section{Discussion}

Elastography is widely used in the diagnosis of liver fibrosis and in the differential diagnosis of liver, breast and thyroid tumors. By evaluating the hardness of the pathological tissue of these organs, the nature and de-

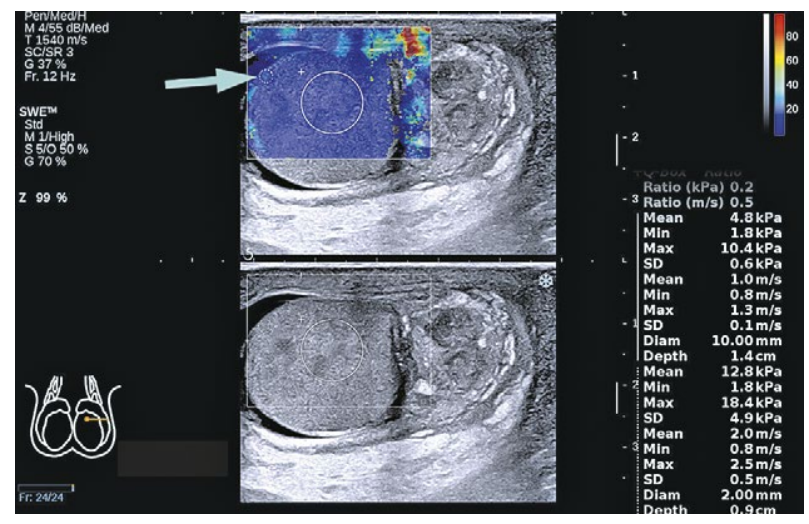

Fig 3. Acute orchid-epididymitis in a 43-year-old patient, SWE examination, transverse scan. The capsule area was blue-green (arrow), Emax value $=18.4 \mathrm{kPa}$

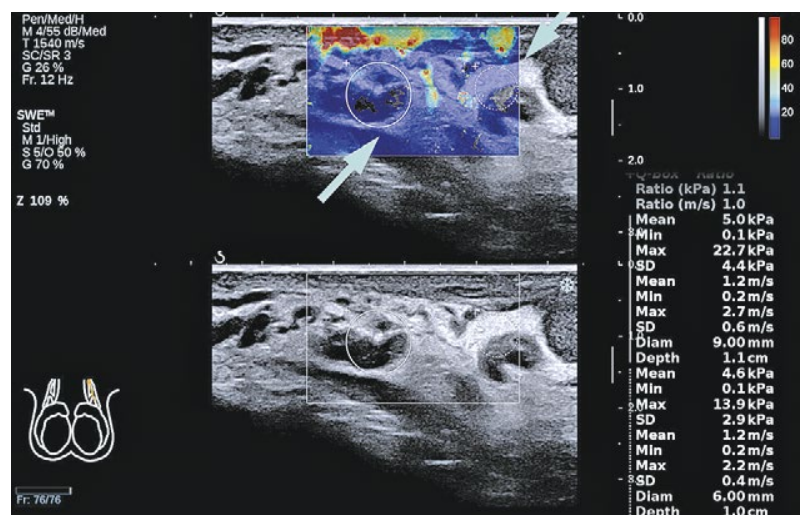

Fig 4. Acute spermatitis in a 28-year-old pacient, SWE examination, longitudinal scan. The spermatic cord was blue-green (arrows) and the Emax value was $22.7 \mathrm{KPa}$

gree of the lesion can be determined [19-21]. Elastography was also applied in testicular pathology, including tumors and spermatogenic function in infertile patients [22-24]. Using Young's modulus, the Emean (mean stiffness) values fit to evaluate the hardness of tissue diffuse lesions [16,25], and the Emax values are most valuable for determining the hardness of focal lesions $[26,27]$.

We found the normal lower spermatic segment as uniform in thickness, straight in shape, soft in texture with SWE uniform in blue and Young's modulus Emax value $11.45 \pm 2.50 \mathrm{kPa}$. A few normal lower spermatic segments

Table II. Interobserver agreement elastography max values independently measured by 2 examiners

\begin{tabular}{lllllll}
\hline & \multicolumn{5}{l}{ Torsion group } & \multicolumn{3}{c}{ Inflammation group } \\
\cline { 2 - 7 } & Testicular capsule & Parenchyma testis & Spermatic cord & Testicular capsule & Parenchyma testis & Spermatic cord \\
\hline Examiner 1 & $138.76 \pm 58.27$ & $12.39 \pm 6.56$ & $166.61 \pm 60.07$ & $16.40 \pm 4.71$ & $9.65 \pm 4.86$ & $14.14 \pm 4.94$ \\
Examiner 2 & $133.47 \pm 60.44$ & $11.77 \pm 4.92$ & $162.94 \pm 61.34$ & $15.74 \pm 5.42$ & $9.16 \pm 3.48$ & $14.26 \pm 5.52$ \\
ICC value & 0.96 & 0.876 & 0.971 & 0.883 & 0.866 & 0.879 \\
\hline
\end{tabular}

ICC: intraclass correlation coefficient 
were tortuous but soft. This tortuous shape is associated with scrotal relaxation and contraction, which is a physiological phenomenon. Acute orchitis, being accompanied by inflammation of the lower spermatic cord, was characterized by swelling and dilatation of blood vessels. The Emax value of Young's modulus in the inflamed lower spermatic cord was significantly lower than that in the torsion spermatic cord but without significant difference compared with normal spermatic cord.

Congenital abnormalities such as abnormal attachment of spermatic tunica vaginalis, excessively long spermatic cord and dysplasia of gubernaculum testis are the anatomical basis of testicular torsion. Deep sleep, violent injury and strenuous exercise can all cause increased scrotal peristalsis and induce excessive testicular rotation to twist the lower spermatic cord. When the torsion of the lower spermatic cord exceeds $180^{\circ}$, it can compress the pampiniform plexus in the lower spermatic cord and block the blood flow in the testis. When the torsion of the lower spermatic segment exceeds $360^{\circ}$, the testicular artery in the lower segment is also compressed and the testicular blood supply is interrupted [9]. The lower spermatic segment twists into a group and forms the "vortex sign" [7], with ischemic swelling and hardening of the tissue and SWE shows the typical "stiff knot sign".

Testicular capsule is composed of visceral layer of testicular tunica vaginalis, white membrane and vascular membrane. The visceral layer and white membrane are tough, and the testicular parenchyma is soft, being mainly composed of seminiferous tubule [7]. Therefore, the shear wave velocity values in the middle of the testicle is different comparing with the testicular border [28]. On the color map of SWE, in the control group, we found the normal testicular capsule to be blue-green and the middle testicular parenchyma as a uniform blue color.

In acute testicular inflammation, testicular tissue is swollen due to inflammatory cells infiltration, but the blood vessels have no microthrombus formation or venous reflux barriers, so no significant increase in pressure in the testis appears. For this reason, we detected no "stiff ring sign" in the testicular capsule. Emax value of Young's modulus in the middle parenchyma of testis in acute orchitis was not significantly different from the value obtained in the torsion group $(\mathrm{p}=0.053)$, therefore, we do not recommend the use of Emax value from the middle parenchyma of testis for differentiation between these pathological situations.

The histopathology of testicular torsion in this group showed microthrombus formation in the lumen of the blood vessels in the testis, edema in the surrounding stroma, and a large number of red blood cell leakage and inflammatory cell infiltration. The parenchyma in the middle of testis was uneven blue or blue-green, and the Emax value of young's modulus was significantly higher than that of normal testis $(p=0.0001)$. Microscopically, the white membrane of the testis is thickened, and the swelling of the testicular tissue can further compress the white membrane and increase its hardness significantly. Therefore, SWE in the testicular capsule showed a yellow-orange or red, and some cases presented a "stiff ring sign", and the Emax value of young's modulus was significantly higher than that of a normal testis or acute orchitis ( $\mathrm{p}=0.000)$.

Testicular torsion is divided into complete and incomplete torsion. In the early stage of testicular incomplete torsion, the blood flow of spermatic vein is blocked but the blood supply of the testicular artery still exists $[29,30]$. We had 2 cases of incomplete torsion in the torsion group in which the lower spermatic segment was twisted, but no "vortex" sign or echogenicity alterations of the testicular parenchyma were found and a small amount of blood flow signals was detected in testicle and twisted spermatic cord. In these cases, the Emax value of the middle parenchyma was only slightly higher but the Emax value of the testicular capsule area and the lower part of the spermatic cord were significantly increased $(>80 \mathrm{Kpa})$, and the lower segment showed "stiff knot" sign. Therefore, when conventional ultrasound cannot clearly determine the existence of torsion, SWE can be used to make a clear diagnosis.

This study has several limitations. Firstly, because it was a retrospective study, the age distribution was uneven, including children and adults. Secondly, for incomplete testicular torsion without "vortex" sign in the lower spermatic segment, Emax values may be different due to the different degrees of spermatic distortion and swelling, and the cut-off value with differential diagnostic value needs to be further discussed by increasing the number of cases.

In conclusion, the Young's modulus Emax of spermatic cord torsional segment, testicular capsule area and middle parenchyma can be significantly increased during testicular torsion. In particular, the "stiff knot" sign in the lower part of the spermatic cord and the "stiff ring sign" in the testicular capsule are typical SWE findings of testicular torsion, which are obviously different from those of acute orchitis.

\section{Conflict of interest: none}

\section{References}

1. Pogorelić Z, Mustapić K, Jukić M, et al. Management of acute scrotum in children: a 25-year single center experience on 558 pediatric patients. Can J Urol 2016;23:8594-8601. 
2. Gaither TW, Copp HL. State appellant cases for testicular torsion: Case review from 1985 to 2015. J Pediatr Urol 2016;12:291.e1-291.e5.

3. Anderson JB, Williamson RC. Testicular torsion in Bristol: A 25-year review. Br J Surg 1988;75:988-992.

4. Oluyemi OY. Torsion of the spermatic cord: An exemplary early presentation. Niger J Clin Pract 2017;20:12061209.

5. Roth B, Giannakis I, Ricklin ME, Thalmann GN, Exadaktylos AK. An Accurate Diagnostic Pathway Helps to Correctly Distinguish Between the Possible Causes of Acute Scrotum. Oman Med J 2018;33:55-60.

6. Howe AS, Vasudevan V, Kongnyuy M, et al. Degree of twisting and duration of symptoms are prognostic factors of testis salvage during episodes of testicular torsion. Transl Androl Urol 2017;6:1159-1166.

7. Kühn AL, Scortegagna E, Nowitzki KM, Kim YH. Ultrasonography of the scrotum in adults. Ultrasonography 2016;35:180-197.

8. Ayvaz OD, Celayir AC, Moralioglu S, et al. Four-year retrospective look for acute scrotal pathologies. North Clin Istanb 2016;2:182-188.

9. Bandarkar AN, Blask AR. Testicular torsion with preserved flow: key sonographic features and value-added approach to diagnosis. Pediatr Radiol 2018;48:735-744.

10. Paltiel HJ, Estrada CR Jr, AlomariAI, et al. Multi-planar dynamic contrast-enhanced ultrasound assessment of blood flow in a rabbit model of testicular torsion. Ultrasound Med Biol 2014;40:361-370.

11. Marcon J, Trottmann M, Stief CG, Clevert DA. CEUS-Use in testicular pathologies. Radiologe 2018;58:572-578.

12. Badea R, Lucan C, Suciu M, Vasile T, Gersak M. Contrast enhanced harmonic ultrasonography for the evaluation of acute scrotal pathology. A pictorial essay. Med Ultrason 2016;18:110-115.

13. Bertolotto M, Muça M, Currò F, Bucci S, Rocher L, Cova MA. Multiparametric US for scrotal diseases. Abdom Radiol (NY) 2018;43:899-917.

14. Ophir J, Céspedes I, Ponnekanti H, Yazdi Y, Li X. Elastography: a quantitative method for imaging the elasticity of biological tissues. Ultrason Imaging 1991;13:111-134.

15. Zemanová M. New Diagnostic Imaging Technique - Shear Wave Elastography. Cesk Slov Oftalmol Fall 2016;72:103110.

16. Turna O, Alis D. A comparative study of shear wave elastography in the evaluation of undescended and retractile testes in a pediatric population. J Med Ultrason 2019;46:231-237.

17. Sun Z, Xie M, Xiang F, et al. Utility of Real-Time Shear Wave Elastography in the Assessment of Testicular Torsion. Plos One 2015; 10:e0138523.
18. Zhang X, Lv F, Tang J. Shear wave elastography (SWE) is reliable method for testicular spermatogenesis evaluation after torsion. Int J Clin Exp Med 2015;8:7089-7097.

19. Schmillevitch J, Chammas MC, Pugliese V, et al. Acoustic radiation force impulse (ARFI) elastography compared with biopsy for evaluating hepatic fibrosis after liver transplantation: a cross-sectional diagnostic study. Sao Paulo Med J 2016;134:513-518.

20. Stoian D, Timar B, Craina M, Bernad E, Petre I, Craciunescu M. Qualitative strain elastography - strain ratio evaluation - an important tool in breast cancer diagnostic. Med Ultrason 2016;18:195-200.

21. Colakoglu B, Yildirim D, Alis D, et al. Elastography in Distinguishing Benign from Malignant Thyroid Nodules. J Clin Imaging Sci 2016;6:51.

22. Goddi A, Sacchi A, Magistretti G, Almolla J, Salvadore M. Real-time tissue elastography for testicular lesion assessment. Eur Radiol 2012;22:721-730.

23. Pozza C, Gianfrilli D, Fattorini G, et al. Diagnostic value of qualitative and strain ratio elastography in the differential diagnosis of non-palpable testicular lesions. Andrology 2016;4:1193-1203.

24. Li M, Du J, Wang ZQ, Li FH. The value of sonoelastography scores and the strain ratio in differential diagnosis of azoospermia. J Urol 2012;188:1861-1866.

25. Shin HJ, Lee YS, Yoon H, et al. Testicular volume and elasticity changes in young children with undescended testes. Med Ultrason 2017;19:380-385.

26. 26.Dobruch-Sobczak K, Zalewska EB, Gumińska A, et al. Diagnostic Performance of Shear Wave Elastography Parameters Alone and in Combination with Conventional B-Mode Ultrasound Parameters for the Characterization of Thyroid Nodules: A Prospective, Dual-Center Study. Ultrasound Med Biol 2016;42:2803-2811.

27. Schäfer FK, Hooley RJ, Ohlinger R, et al. ShearWave ${ }^{\mathrm{TM}}$ Elastography BE1 multinational breast study: additional SWE ${ }^{\mathrm{TM}}$ features support potential to downgrade BIRADS®-3 lesions. Ultraschall Med 2013;34:254-259.

28. Trottmann M, Marcon J, D'Anastasi M, et al. Shear-wave elastography of the testis in the healthy man - determination of standard values. Clin Hemorheol Microcirc 2016;62:273-281.

29. Xue E, Guo J, Huang M, Zhang Y, Chen S, Liang R. Effects of gradual low-flow reperfusion postconditioning on ischemia-reperfusion injury involving incomplete testicular torsion in rabbits. J Med Ultrason 2015;2:207-214.

30. Patoulias D, Farmakis K, Kalogirou M, Patoulias I. Transient testicular torsion: from early diagnosis to appropriate therapeutic intervention (a prospective clinical study). Folia Med Cracov 2017;57:53-62. 